\title{
Textos sobre medicina y salud en la prensa diaria. Su potencial utilidad para la adquisición de competencias de grado en Medicina y Ciencias de la Salud.
}

\section{Texts about medicine and health in the daily press. Its potential utility for the acquisition of undergraduate competencies in Medicine and Health Sciences.}

\author{
María González-García ${ }^{1}$, Begoña Cantabrana ${ }^{2}$, Agustín Hidalgo $^{3^{*}}$ \\ 1 Diplomada en Enfermería, Hospital Universitario Central de Asturias. Instituto de Investigación Sanitaria \\ de Asturias. mariagonzalezgarcia@gmail.com. ORCID: https://orcid.org/0000-0002-1734-9562 \\ 2 Profesora Titular. Área de Farmacología. Universidad de Oviedo. Instituto Universitario de Oncología. \\ Obra Social CajAstur. Instituto de Investigación Sanitaria de Asturias. bego@uniovi.es. ORCID: \\ https://orcid.org/0000-0002-9436-9487 \\ 3 Catedrático de Universidad. Área de Farmacología. Universidad de Oviedo. Instituto Universitario de \\ Oncología. Obra Social CajAstur. Instituto de Investigación Sanitaria de Asturias. hidalgo@uniovi.es. \\ ORCID: https://orcid.org/0000-0003-4177-0928 \\ * Correspondencia: hidalgo@uniovi.es
}

Recibido: 16 de junio de 2020; Aceptado: 6 de julio de 2020; Publicado: 7 de julio de 2020

Resumen: Los estudios sobre textos periodísticos relacionados con la salud han adquirido una importancia creciente por lo que hemos estudiado la distribución temática de noticias de prensa sobre medicina y salud publicadas en el periodo 2001-2016, y explorado su utilidad formativa potencial. Se realizó un estudio descriptico de un conjunto de noticias de prensa sobre medicina y salud publicadas en el diario El País a lo largo de 16 años que están recogidos en la colección CONPRE-Oviedo. La distribución de las noticias se realizó de acuerdo a la Clasificación Internacional de Enfermedades (CIE-10) o por su contenido temático cuando no se ajustaba a CIE-10. También se identificaron una serie de competencias generales de la Orden ECI 332/2008 que pueden ser iniciadas mediante utilización docente de textos periodísticos. Se seleccionaron 1828 noticias (media: 114,3/año). El 63\% pueden clasificarse mediante CIE-10, siendo las más frecuentes las categorías 1: Ciertas enfermedades infecciosas y parasitarias; 15: Embarazo, parto y puerperio; 10: Enfermedades del aparato respiratorio y 2: Neoplasias. De las no clasificadas según CIE-10, las más frecuentes se relacionaron con gestión sanitaria y salud pública. Se detectaron dos patrones de aparición de noticias: uno continuo, a lo largo de los 16 años, y otro incidental. Se han identificado 34 competencias a cuya adquisición pueden contribuir los textos periodísticos y se aportan algunos ejemplos. En conclusión: a) en la prensa escrita se encuentran textos referidos a la mayor parte de las enfermedades recogidas en la CIE-10, lo que puede contribuir a la educación para la salud. El tratamiento periodístico de esta información puede ser incidental o mantenido en el tiempo según la naturaleza de las enfermedades; b) la utilización docente de textos periodísticos puede contribuir a la adquisición de competencias transversales en la formación de grado en Medicina y otras Ciencias de la Salud.

Palabras clave: Textos periodísticos. Clasificación Internacional de enfermedades. Cultura sanitaria. Hábitos de vida saludable. Educación Médica. Competencias transversales de grado. 


\begin{abstract}
Studies on journalistic texts related to health have become increasingly important, so we have studied the thematic distribution of press news on medicine and health published in the period 2001-2016, and explored its potential formative usefulness. A descriptive study was carried out of a set of news reports on medicine and health published in the newspaper El País over the course of 16 years that are included in the CONPRE-Oviedo collection. The news was distributed according to the International Classification of Diseases (ICD-10) or for its thematic content when it did not comply with ICD-10. A series of general competences of Order ECI 332/2008 were also identified that can be initiated through the educational use of journalistic texts.1828 news items were selected (mean: 114.3 / year). 63\% can be classified by ICD-10, the most frequent being categories 1: Certain infectious and parasitic diseases; 15: Pregnancy, childbirth and the puerperium; 10: Diseases of the respiratory system and 2: Neoplasms. Of those not classified according to ICD-10, the most frequent were related to health management and public health. Two patterns of news appearance were detected: a continuous one, throughout the 16 years, and an incidental one. 34 competences to which journalistic texts can contribute have been identified and some examples are provided. In conclusion: a) in the written press there are texts referring to most of the diseases included in the ICD-10, which can contribute to health education. The journalistic treatment of this information may be incidental or maintained over time depending on the nature of the diseases; $b$ ) The teaching use of journalistic texts can contribute to the acquisition of transversal competences in undergraduate training in Medicine and other Health Sciences.
\end{abstract}

Keywords: Journalistic texts. International Classification of diseases. Health culture. Healthy life habits. Medical education. Transversal undergraduate competences.

\title{
1. Introducción
}

La divulgación científica se remonta al siglo XVII, siendo tras la Segunda Guerra Mundial cuando se produce una mayor proyección en la sociedad (1-2). El interés por explicar al mundo lo que ocurría, así como el derecho de los ciudadanos a saber, compartir y participar en la toma de decisiones, convirtió a los medios de comunicación en un puente entre los centros de investigación y la población (3-5). A partir de los últimos veinte años del siglo pasado, el crecimiento continuo de la producción científica española, específicamente de la medicina clínica ha favorecido (6) la especialización de la información de la prensa, haciendo que se creasen secciones específicas sobre ciencia y medicina $(3,7)$. Así fue como El País, diario de mayor tirada nacional, comenzó a editar en 1986 el suplemento Futuro dedicado a la ciencia, la salud y la tecnología $(3,5)$.

El incremento del interés por la ciencia producido en los últimos años, especialmente por su repercusión en la medicina y la salud, puede ser un factor importante para facilitar a la población información que mejore su cultura en medicina y salud. Los medios de comunicación, en concreto la prensa escrita, son un vehículo de fácil acceso y permanente en el tiempo $(8,9)$, por lo que pueden ocupar un lugar relevante en la alfabetización médica de la población $(2,10) \mathrm{y}$, por lo tanto, en el empoderamiento para el cuidado respecto de su propia salud. Por otra parte, se asume que el público no experto adquiere la 
mayor parte del conocimiento científico y sanitario a través de los medios de comunicación (11), apropiación que también ha sido puesta de manifiesto en estudiantes de Medicina $(12,13)$.

En nuestro país, diferentes estudios han hecho análisis puntuales de textos periodísticos relacionados con la medicina y la salud (14-16) y existen algunos estudios longitudinales que permanecen abiertos desde hace décadas (17). También es conocido que los medios pueden ser útiles en determinadas campañas de salud pública orientadas a la adquisición de hábitos saludables $(18,19)$ o de participación en campañas de vacunación $(20,21)$. En un estudio previo hemos descrito la colección de noticias CONPRE-Oviedo (2001-2016) de textos periodísticos relacionados, entre otros aspectos, con ciencia, medicina y medicamentos $(22,23)$.

En este artículo describimos la distribución de las noticias sobre medicina y salud contenidas en la colección y exploramos las competencias formativas que los textos periodísticos pueden contribuir a su adquisición por los estudiantes de Medicina.

\section{Métodos}

Se planteó un estudio descriptivo transversal de un conjunto de textos periodísticos pertenecientes a la colección CONPRE-Oviedo elaborada a lo largo de 16 años (2001 a 2016) utilizando la edición impresa del diario El País (el de mayor tirada de la prensa española) siguiendo los siguientes criterios: a) el título debe aludir a términos de interés para la colección, b) tener un autor identificable, c) estar basada en hechos reales, d) tener entidad narrativa y e) contener una historia completa. No se han considerado las noticias procedentes de agencias sin un autor identificable (22).

En primer lugar, se identificaron las noticias relacionadas con medicina y salud contenidas en la colección. Posteriormente, para analizar la distribución de las noticias seleccionadas correspondientes al apartado de medicina y salud de la antedicha colección se utilizó la Clasificación Internacional de Enfermedades (CIE-10) (24). Para la adscripción de las noticias a las diferentes categorías de la CIE-10, han participado al menos dos de los autores del artículo, resolviendo los desacuerdos por consenso con el tercer autor. Cuando no se ajustaron a las categorías de la CIE-10, se agruparon por su contenido temático.

Para la identificación de las competencias que, tentativamente, pueden contribuir a adquirir los textos literarios, hemos analizado las competencias transversales descritas en la Orden ECI/332 de 13 de febrero de 2008 (25) por la que se establecen los requisitos para la verificación de los títulos de médico por la Agencia Nacional de Evaluación de la Calidad y Acreditación, identificando aquellas relacionadas con valores profesionales, habilidades de comunicación, salud pública, fundamentos científicos de la medicina y análisis crítico y de investigación que pueden iniciarse mediante el análisis de textos periodísticos. Por último, hemos identificado, entre los textos recogidos en la colección CONPRE-Oviedo, algunos que pueden apoyar esta utilidad formativa potencial en estudiantes del grado en Medicina. 
Análisis de datos. Se ha realizado una distribución de frecuencias mostrando los datos como media \pm error estándar de la media, valores absolutos y/o relativos dependiendo de la variable estudiada.

Por otra parte, han sido identificadas algunas noticias relacionadas tanto con temas incidentales como con temas mantenidos a lo largo de la colección a partir de los cuales se ha analizado su potencial formativo en estudiantes del grado en Medicina.

\section{Resultados}

\subsection{Textos periodísticos sobre medicina y salud}

La colección de textos periodísticos está formada por 6494 noticias de las que 1828 están relacionadas con medicina y salud. De ellas, más de la mitad ( $\mathrm{n}=1151 ; 63 \%)$ se clasificaron según la CIE-10 mientras que el $35,3 \%$ restante $(n=677)$ se agruparon en diferentes temas según se puede observar en la Tabla 1, que también muestra que la mayoría de las categorías están presentes en la colección de noticias. Por otra parte, la media de noticias por año fue de 114,25.

La frecuencia de textos periodísticos, según la clasificación CIE-10, fue variable, siendo los cinco apartados que más se repiten: Ciertas enfermedades infecciosas y parasitarias (categoría 1) > Embarazo, parto y puerperio (categoría 15) > Enfermedades del aparato respiratorio (categoría 10) > Neoplasias (categoría 2) > Factores que influyen en el estado de salud y contacto con los servicios sanitarios (categoría 21) (Tabla 1).

Tabla 1. Distribución de noticias clasificadas en el apartado de medicina y salud. Cuando no se ha podido clasificar según la CIE-10, se ha hecho una agrupación temática.

\begin{tabular}{|l|c|c|}
\hline \multicolumn{1}{|c|}{ CIE-10 } & $\mathbf{n}$ & $\mathbf{\%}$ \\
\hline 1. Ciertas enfermedades infecciosas y parasitarias & 249 & 21,6 \\
\hline 2. Neoplasias & 127 & 11,0 \\
\hline $\begin{array}{l}\text { 3. Enfermedades de la sangre y órganos hematopoyéticos y trastornos } \\
\text { que afectan al mecanismo inmunológico }\end{array}$ & 1 & 0,1 \\
\hline 4. Enfermedades endocrinas, nutricionales y metabólicas & 58 & 5,0 \\
\hline 5. Trastornos mentales y del comportamiento & 76 & 6,6 \\
\hline 6. Enfermedades del Sistema Nervioso & 52 & 4,5 \\
\hline 7. Enfermedades del ojo y sus anexos & 4 & 0,3 \\
\hline 8. Enfermedades del oído & 0 & 0 \\
\hline 9. Enfermedades del aparato circulatorio & 46 & 4,0 \\
\hline 10. Enfermedades del aparato respiratorio & 146 & 12,7 \\
\hline 11. Enfermedades del aparato digestivo & 5 & 0,4 \\
\hline 12. Enfermedades de la piel y del tejido subcutáneo & 5 & 0,4 \\
\hline 13. Enfermedades del aparato musculoesquelético y del tejido conectivo & 12 & 1 \\
\hline 14. Enfermedades del aparato genitourinario & 11 & 1 \\
\hline 15. Embarazo, parto y puerperio & 175 & 15,2 \\
\hline 16. Ciertas afecciones originadas en el periodo perinatal & 4 & 0,3 \\
\hline $\begin{array}{l}\text { 17. Malformaciones congénitas, deformidades y anomalías } \\
\text { cromosómicas }\end{array}$ & 11 & 1,0 \\
\hline 18. Síntomas, signos y resultados anormales de pruebas & 2 & 0,2 \\
\hline
\end{tabular}




\begin{tabular}{|l|c|c|}
\hline \multicolumn{1}{|c|}{ complementarias, clasificadas bajo otro concepto } & & \\
\hline $\begin{array}{l}\text { 19. Lesiones, envenenamientos y otras consecuencias de causas } \\
\text { externas }\end{array}$ & 7 & 0,6 \\
\hline 20. Causas externas de morbilidad en el estado de salud y contacto con los & 39 & 3,4 \\
\hline $\begin{array}{l}\text { 21. Factores que influyen en } \\
\text { servicios sanitarios }\end{array}$ & 10,5 \\
\hline Noticias no clasificadas en Cie-10 & $\mathrm{N}$ & $\%$ \\
\hline Gestión sanitaria & 258 & 38,1 \\
\hline Salud Publica & 157 & 23,2 \\
\hline El final de la vida & 120 & 17,7 \\
\hline Profesionalismo & 40 & 5,9 \\
\hline Relación médico/paciente & 35 & 5,2 \\
\hline Iatrogenia & 29 & 4,3 \\
\hline Envejecimiento & 14 & 2,1 \\
\hline Técnicas & 9 & 1,3 \\
\hline Enfermedades raras & 8 & 1,2 \\
\hline Ética & 7 & 1,0 \\
\hline Otros & 13 & 1,9 \\
\hline
\end{tabular}

Cuando observamos la evolución anual de los textos periodísticos, encontramos que los correspondientes a las categorías 2, 4, 5, 6, 9 y 21 tienen una presencia continua a lo largo de los años. Por el contrario, otros están muy concentradas en unos pocos años, como las enfermedades del aparato respiratorio que, de un total de 146 noticias, sólo en el año 2009 se recogieron 109 relacionadas con la epidemia de Gripe A o H1N1. De forma ilustrativa, la Figura 1 muestra la evolución de los textos de algunos apartados de las categorías recogidas en la colección.

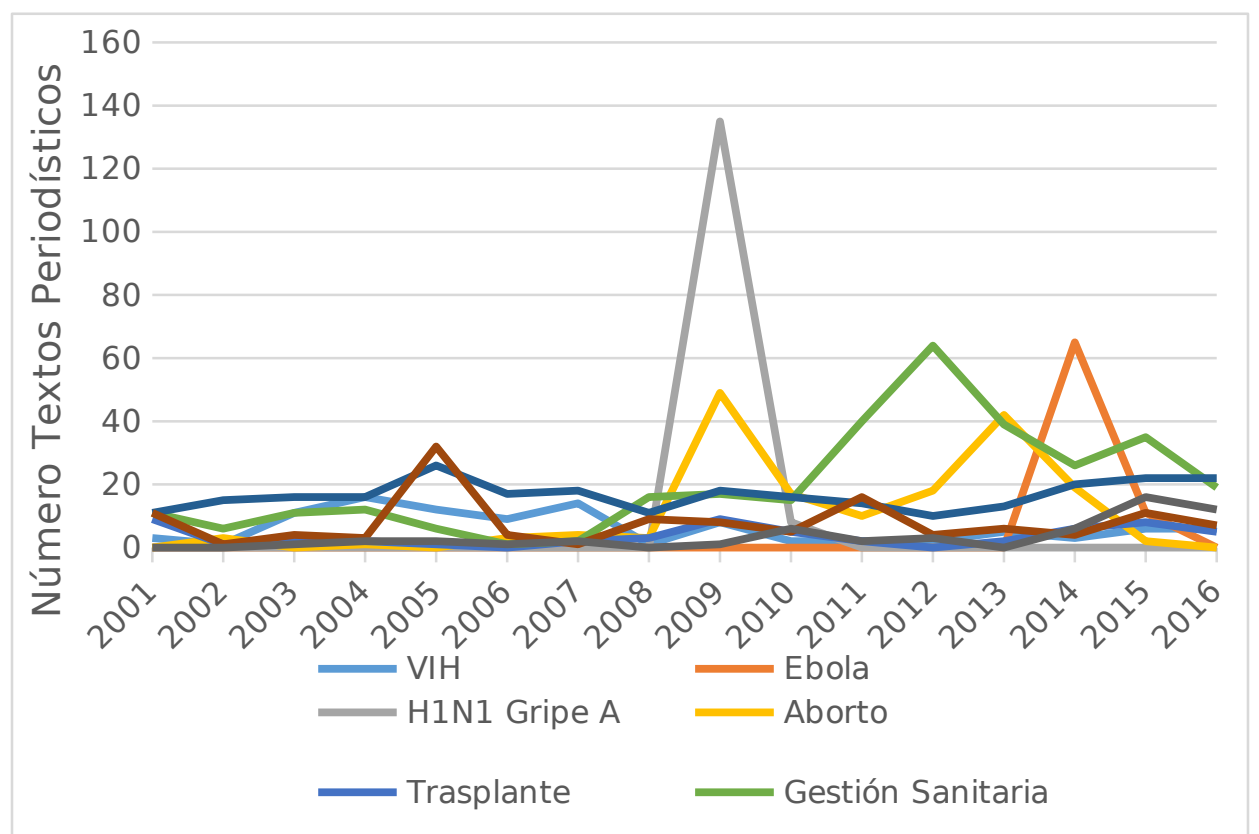

Figura 1. Evolución temporal de una serie de textos periodísticos durante el período 2001-2016. La figura muestra los dos patrones de evolución: el incidental (en picos) y continuo (plano, con pequeñas oscilaciones). 
Cuando identificamos las enfermedades o prácticas médicas referidas con más frecuencia en la prensa diaria y consideramos todos los textos referidas a las mismas, observamos que el aborto, la gripe H1N1 o la infección por el VIH son las tres más abundantes; de hecho, referidas al VIH encontramos una media de 5,6 textos/año. En otros casos, la incidencia ha sido más puntual, como para el Ébola o el virus Zika, en los que las noticias se han concentrado en los años 2014 y 2016 respectivamente.

En la Tabla 1 se indican también los 10 temas más citados que no se relacionan con los de la clasificación CIE-10. De ellos, la Gestión Sanitaria (política sanitaria, gestión clínica y sistema nacional de salud) es la más frecuente (40\%) junto a las referidas a la Salud Pública y noticias relacionadas con el final de la vida (eutanasia, muerte digna y cuidados paliativos). Los textos periodísticos atribuibles al profesionalismo sanitario y a la relación médico-enfermo tienen una menor presencia en los medios.

\subsection{Posible valor formativo}

La aproximación al potencial formativo admite un doble planteamiento. Por una parte, es la necesidad de una relación del contenido del texto periodístico con el cuerpo doctrinal de la medicina, tanto por su proximidad a las competencias específicas y/o huérfanas atribuidas a la formación de grado. Por otra parte, la identificación de la forma adecuada para transformar el texto periodístico en material didáctico.

A modo de aproximación al tema, hemos analizado las competencias transversales contenidas en apartado 3 del Anexo de la Orden ECI/332 del 2008 para la verificación de los estudios de grado de medicina. De los 7 apartados que incluye hemos seleccionado 6, dos de los cuales (Manejo de la información y Habilidades de comunicación) los hemos refundido en uno bajo la denominación de Habilidades de comunicación. A cada una de estas categorías le hemos asignado una serie de competencias cuya adquisición puede ser iniciada o facilitada mediante análisis de textos periodísticos. En parte estas competencias están contenidas en la orden ECI y otras las hemos formulado para este artículo. Estas competencias, en número de 34, se encuentran distribuidas en las siguientes categorías de la Orden ECI antedicha: valores profesionales, habilidades de comunicación, salud pública, fundamentos científicos de la medicina y análisis crítico y de investigación (Tabla 2). 
Tabla 2. Relación de competencias a cuya iniciación/adquisición puede contribuir, al menos parcialmente, el análisis de textos periodísticos.

\begin{tabular}{|c|c|}
\hline Área temática & $\begin{array}{l}\text { Competencias (mediante los textos periodísticos, los } \\
\text { estudiantes pueden ser expuestos a situaciones que les } \\
\text { permitan aproximaciones a...) }\end{array}$ \\
\hline $\begin{array}{l}\text { Valores } \\
\text { profesionales }\end{array}$ & $\begin{array}{l}\text { 1.Valores éticos de la profesión y del profesionalismo } \\
\text { 2. Justicia social en la práctica médica } \\
\text { 3. Valorar el papel de la cultura y la creencia religiosa en la } \\
\text { salud } \\
\text { 4. Motivación social de los estudiantes } \\
\text { 5. El valor social del individuo a lo largo de la vida } \\
\text { 6. Adquisición de terminología médica }\end{array}$ \\
\hline $\begin{array}{l}\text { Habilidades de } \\
\text { comunicación }\end{array}$ & $\begin{array}{l}\text { 7. Utilizar tecnologías de la información y comunicación } \\
\text { 8. Obtener y sintetizar información pertinente en medicina } \\
\text { 9. La confidencialidad de los datos de los estudios clínicos } \\
\text { 10. Analizar textos de diferentes géneros periodísticas } \\
\text { 11. Utilizar la información para resolver problemas } \\
\text { 12. Redactar informes y ensayos sobre temas de medicina y } \\
\text { salud } \\
\text { 13. Ensayar habilidades de comunicación oral y escrita. } \\
\text { 14. Desarrollar habilidades de comunicación interpersonal } \\
\text { 15. Fomento de una actitud discursiva crítica }\end{array}$ \\
\hline Salud Pública & $\begin{array}{l}\text { 16. Reconocer y valorar los determinantes de salud pública } \\
\text { (genéticos, demográficos, sociales, de estilos de vida...) } \\
\text { 17. Conocer estrategias de promoción de hábitos de vida } \\
\text { saludable tanto a nivel individual como colectivo } \\
\text { 18. Ponderar el valor de los datos epidemiológicos en la toma } \\
\text { de decisiones } \\
\text { 19. Conocer organizaciones y agencias nacionales e } \\
\text { internacionales relacionadas con la salud }\end{array}$ \\
\hline $\begin{array}{l}\text { Fundamentos } \\
\text { científicos de la } \\
\text { medicina }\end{array}$ & $\begin{array}{l}\text { 20. Fundamentos de filosofía de la ciencia } \\
\text { 21. Revoluciones tecno-científicas y su repercusión en salud } \\
\text { 22. Limitaciones de la elaboración científica en medicina } \\
\text { 23. Comportamiento humano y sus condicionantes } \\
\text { 24. Aproximación a riesgos médicos y sus agentes causales } \\
\text { 25. Determinantes de salud y enfermedad } \\
\text { 26. El individuo como sistema abierto: relación con el entorno y } \\
\text { su repercusión en salud } \\
\text { 27. Responsabilidades sociales del uso de medicamentos y } \\
\text { otras sustancias químicas }\end{array}$ \\
\hline $\begin{array}{l}\text { Análisis crítico y } \\
\text { de investigación }\end{array}$ & $\begin{array}{l}\text { 28. Fomento de un escepticismo constructivo } \\
\text { 29. Comprender los límites del conocimiento científico en } \\
\text { medicina } \\
\text { 30. Adquirir información para resolver problemas } \\
\text { 31. Entrenamiento en discurso lógico } \\
\text { 32. Concepto de certeza en investigación científica } \\
\text { 33. Asunción de los principios de sociología de la ciencia y de la } \\
\text { medicina como componentes de la investigación científica y de } \\
\text { la comunicación en salud } \\
\text { 34. Críticas de las diatribas que ofrecen los medios a propósitos } \\
\text { de procedimientos médicos }\end{array}$ \\
\hline
\end{tabular}

Con el fin de explorar si los textos periodísticos pueden adaptarse en alguna medida a los temas habituales en la formación médica, hemos diseñado la Tabla 3 en la que se muestran varios textos periodísticos, su relación con aspectos de la enseñanza de la medicina y las competencias de la Tabla 2 con cuya adquisición estarían relacionadas. 
Tabla 3. Ejemplos de la potencialidad utilidad de los textos periodísticos para la adquisición de competencias formativas de grado en Medicina. Los textos fueron publicados en la fecha que se indica en el diario El País.

\begin{tabular}{|c|c|c|}
\hline $\begin{array}{c}\text { Texto } \\
\text { Periodístico }\end{array}$ & 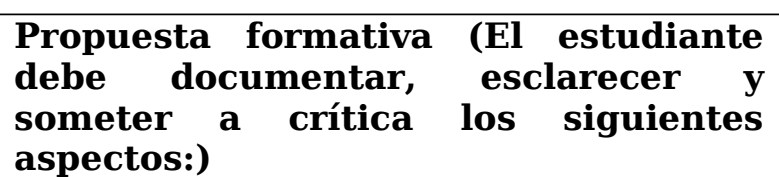 & $\begin{array}{lr}\text { Competencias } & \text { que } \\
\text { practica } & \text { (Área } \\
\text { temática y } & \text { No } \\
\text { competencia } & \text { de }\end{array}$ \\
\hline $\begin{array}{l}\text { Benito } \\
\text { Descubierto un } \\
\text { gen } \\
\text { responsable de } \\
\text { los cánceres de } \\
\text { próstata más } \\
\text { agresivos. } \\
2015\end{array}$ & $\begin{array}{l}\text { - Mortalidad por cáncer en España y } \\
\text { Europa } \\
\text { - Factores predisponentes al cáncer } \\
\text { proliferación celular } \\
\text { - Colaboración molecular en las señales } \\
\text { oncogénicas } \\
\text { - Concepto de "ratón avatar" y su papel } \\
\text { en la medicina personalizada" } \\
\text { - Concepto de Resistencia a agentes } \\
\text { antineoplásicos }\end{array}$ & $\begin{array}{l}\text { Salud Pública: } 16-18 \\
\text { Fundamentos científicos } \\
\text { de la medicina: } 20-26 \\
\text { Análisis crítico y de } \\
\text { investigación: } 28-33\end{array}$ \\
\hline $\begin{array}{l}\text { Méndez M y } \\
\text { Morán C. } \\
\text { Indemnización } \\
\text { para las } \\
\text { víctimas de la } \\
\text { talidomida } 40 \\
\text { años después. } \\
\text { 23-3-2010 }\end{array}$ & $\begin{array}{l}\text { Cronología de una epidemia } \\
\text { "evitable" } \\
\text { - Concepto de teratogenia e } \\
\text { implicación del medicamento Talidomida } \\
\text { - Responsabilidad del productor y de la } \\
\text { administración. Justicia social } \\
\text { Ética de la investigación y del } \\
\text { desarrollo de medicamentos. } \\
\text { Ética profesional } \\
\text { - Estigma social }\end{array}$ & $\begin{array}{l}\text { Valores Profesionales: } \\
1,2,5 \text {. } \\
\text { Análisis crítico y de } \\
\text { investigación: } 28,31,33 \text {. }\end{array}$ \\
\hline $\begin{array}{l}\text { Benito E. } \\
\text { Los parados se } \\
\text { drogan más. } \\
27-1-2011\end{array}$ & $\begin{array}{l}\text { - Epidemiología de la drogadicción } \\
\text { - Consumo de drogas en el ámbito } \\
\text { laboral } \\
\text { • ¿Qué tipo de trabajo incita más a la } \\
\text { drogadicción? } \\
\text { ¿ ¿La categoría profesional condiciona } \\
\text { la drogadicción? } \\
\text { - ¿Hay diferencias de género? } \\
\text { cCuáles son las drogas más } \\
\text { consumidas? }\end{array}$ & $\begin{array}{l}\text { Salud Pública: } 16-19 \\
\text { Fundamentos científicos } \\
\text { de la medicina: } 22-27\end{array}$ \\
\hline $\begin{array}{l}\text { Monge Y. } \\
\text { EEUU pide } \\
\text { perdón a a } \\
\text { Guatemala por } \\
\text { experimentar } \\
\text { con pacientes y } \\
\text { presos. 2-10- } \\
2010\end{array}$ & $\begin{array}{l}\text { Ética de la investigación en medicina } \\
\text { clínica } \\
\text { - Conocer el código deontológico } \\
\text { médico } \\
\text { - Papel del consentimiento informado } \\
\text { Papel de los CEIm. ¿Autorizarían este } \\
\text { estudio? } \\
\text { investigación con medicamentos } \\
\text { - Documentar estudios históricos faltos } \\
\text { de ética }\end{array}$ & $\begin{array}{l}\text { Valores profesionales: } \\
1,2,4,5 \\
\text { Fundamentos científicos } \\
\text { de la medicina: } 21 \\
\text { Análisis crítico y de } \\
\text { investigación: } \\
27,28,32,34 .\end{array}$ \\
\hline $\begin{array}{l}\text { Benito E. } \\
\text { Los enfermos } \\
\text { "raros" toman } \\
\text { las riendas. 23- } \\
2-2014\end{array}$ & $\begin{array}{l}\text { - Concepto de "Enfermedad rara" y su } \\
\text { dimensión. } \\
\text { - Causas más frecuentes } \\
\text { "enfermedades raras" } \\
\text { - Razones del "abandono" de las } \\
\text { enfermedades raras. } \\
\text { - Papel de la autofinanciación y } \\
\text { micromecenazgo } \\
\text { - Relevancia del consejo genético y } \\
\text { terapia génica }\end{array}$ & $\begin{array}{l}\text { Valores profesionales: } 2 \\
\text { Salud Pública: } 16,19 \\
\text { Fundamentos científicos } \\
\text { de la medicina: } 20,21,25 \\
\text { Análisis crítico y de } \\
\text { investigación: } 28,32 \text {. }\end{array}$ \\
\hline
\end{tabular}




\begin{tabular}{|l|l|l|}
\hline & $\begin{array}{l}\bullet \text { Concepto y contribución de la } \\
\text { medicina molecular } \\
\bullet \quad \text { Identificar algunos casos de éxito de } \\
\text { terapia génica. }\end{array}$ \\
\hline${ }^{1}$ El número de competencia se refiere a las contenidas en la tabla 2. \\
\hline
\end{tabular}

A modo de ejemplo, de entre los temas recogidos en la colección CONPRE-Oviedo hemos seleccionado 5 textos periodísticos, referidos a otras tantas situaciones que tienen que ver con asuntos relacionados con la medicina y la salud, que pueden ilustrar la adecuación de estas fuentes para la adquisición de competencias. Uno de estos textos alude al descubrimiento de un gen que puede tener alguna responsabilidad en la proliferación celular en el cáncer de próstata agresivo y que responde mal o no responde a tratamiento (28). La propuesta de un seminario sobre la base de esta noticia puede ayudar a los estudiantes a documentar aspectos relacionados con su formación en Salud Pública, en Oncología, en Biología Celular y Tumoral y, también, a plantear cuestiones que tienen que ver con los fundamentos científicos de la medicina y con la forma de elaborar ciencia.

Otro de los temas seleccionados facilita el análisis de algunos valores profesionales relacionados con la implicación en estudios clínicos, y otros más propios del análisis crítico de la investigación, estando presente en ambos casos los componentes éticos. Si bien este tema, la epidemia de focomelia provocada por la Talidomida (29), forma parte del cuerpo doctrinal de alguna asignatura, no es menos cierto que tiene un valor transversal a muchas disciplinas por sus implicaciones éticas, morales y sociales, más allá de las estrictas connotaciones biomédicas.

El tercero de los temas alude a una cuestión asimismo transversal que puede enfocarse desde la Farmacología Clínica o Social, la Epidemiología, la Psicología, la Psiquiatría, la Sociología, etc. Se trata de la relación entre el consumo de medicamentos y la actividad laboral con una aparente mayor incidencia en los desempleados (30), lo que permite aproximarnos al ámbito de la drogadicción, a la distribución del consumo, a la existencia o no de diferencias de género o a los medicamentos más consumidos con tal fin.

En ocasiones, surgen informaciones que sacan a la luz comportamientos científicos que considerábamos olvidados. Es el caso que presenta el cuarto de los textos literarios seleccionados: la realización de estudios, abalados por un Estado, en un país tercero sin respetar los principios de la investigación clínica ni la ética de la investigación (31). Consideramos que el texto es adecuado para explorar algunos valores de la profesión médica además de los principios de la investigación clínica y analizar su regulación.

El último texto periodístico (32) propuesto se hace eco tanto de la realidad que afecta a enfermos de patologías de baja prevalencia como de una práctica que está creciendo en todos los países y que traduce el empoderamiento social en la toma de decisiones relacionada con los problemas de investigación en salud, como son las enfermedades raras.

Consideramos que la mejor estrategia formativa es el diseño de seminarios en el que los estudiantes en grupos de 3-4 aborden el 
desarrollo de los temas propuestos por el profesorado o por los mismos alumnos. Como se indica en la Tabla 3, los estudiantes deben documentar y dimensionar el tema, dilucidar los diferentes aspectos que estén incluidos en el mismo y someterlos a crítica, procurando fomentar el escepticismo constructivo propio de la ciencia, y extraer las conclusiones que sean pertinentes. A lo largo de este proceso de corroborar y dimensionar la información facilitada, los estudiantes ejercitarán las competencias relacionadas con las habilidades documentación recogidas en la Tabla 2.

\section{Discusión}

El hecho de que la colección CONPRE-Oviedo (2001-2016) contenga más de 100 textos periodísticos al año relacionadas con medicina y salud sugiere que su presencia en los medios de comunicación ha sido continua durante el periodo de observación. A lo largo de los 16 años, el número de textos periodísticos ha sido creciente, acentuándose a partir de 2011, lo que sugiere que estos temas se tratan de forma cotidiana y pueden contribuir a generar interés, apropiación ciudadana de sus temas y a la creación de una cultura de salud (8).

A la vista de la distribución de los temas contenidos en las noticias y de su frecuencia en el diario, podemos considerar que hay cuestiones transversales con presencia continua a lo largo del periodo de estudio (tales como la salud pública, las enfermedades infecciosas o las neoplasias) y otros que tienen una presencia incidental condicionada por la aparición abrupta de un problema periodístico y socialmente relevante (p.e. la incidencia de la Gripe A en 2009 o del Ébola en 2014).

Los temas que han constituido la actualidad biosanitaria de la prensa diaria en los últimos 16 años no difieren demasiado de los años anteriores $(9,17)$. La gestión sanitaria, la salud pública, las enfermedades infecciosas o los problemas éticos que se plantean con actuaciones al principio y al final de la vida, son temas que se han identificado a lo largo del periodo de seguimiento y con los que la población puede sentirse identificada.

De hecho, de las 8 enfermedades más presentes en el diario de referencia, clasificadas según la CIE-10, 3 fueron epidemias, la Gripe A, el Ébola y el Zika. Sobre todo, la primera de ellas generó una elevada atracción periodística, relatando el día a día de la epidemia, así como su tratamiento mediante Oseltamivir ${ }^{\circledR}$ y la prevención mediante la vacunación, generando a su vez miedo en la población $(14,26)$.

Por otra parte, como era de esperar, la distribución de textos periodísticos sigue un patrón más próximo a la realidad social que a la realidad clínica dado que los problemas de gestión sanitaria y otros aspectos transversales tienen una presencia muy elevada entre los textos seleccionados. Esto es particularmente relevante porque afecta a dos aspectos que consideramos críticos: la viabilidad de los sistemas públicos de salud y a la educación sanitaria. Además, los textos referidos a enfermedades recogidas en la clasificación CIE-10, no se corresponden con la realidad asistencial dado que, mientras en la prensa las noticias sobre salud más frecuentes se refieren a enfermedades infecciosas y parasitarias, embarazo, infecciones 
respiratorias y neoplasias, las causas de morbilidad y mortalidad más frecuentes registradas en nuestro país, según el Instituto Nacional de Estadística de 2017, son las enfermedades del sistema circulatorio, seguidas de los tumores y las patologías respiratorias (27). A pesar de ello, consideramos que el papel de la prensa es importante para la creación y apropiación social de una cultura médica y sanitaria, así como para la adquisición de hábitos de vida saludables, y ha sido considerado trascendente en las campañas de inducción de hábitos saludables, como combatir el tabaquismo o el alcoholismo $(18,19)$ o promover la participación en campañas de vacunación y/o la creación de una conciencia crítica en torno a las mismas $(20,21)$.

También, los textos periodísticos pueden utilizarse como material didáctico complementario a las estrategias formativas recogidas en las memorias verificadas de los grados, para aproximar a los estudiantes a la dimensión social de la enfermedad y de la medicina y exponerlos a cuestiones que requieren un tratamiento más amplio (y potencialmente diferenciado) del que cabe en los programas formativos oficiales.

Consideramos que los textos periodísticos seleccionados pueden ser utilizados de forma complementaria a aquellos que cada profesor considere adecuados para la impartición de la materia sobre la que tienen responsabilidad educativa. Al analizar los textos propuestos, documentarlos, ponerlos en el pertinente contexto temporal, científico, social, etc., los estudiantes pueden iniciar o completar la adquisición de competencias relacionadas con las habilidades de comunicación, los valores profesionales, la salud pública o los fundamentos y el análisis crítico de problemas de investigación y su dimensión social. La propuesta que hacemos en este artículo es complementaria de las realizadas en otros trabajos previos en los que hemos analizado la utilidad de los textos periodísticos para la adquisición de lenguaje médico (13) o el posicionamiento de los estudiantes sobre la cultura científica (12) y hemos propuesto que potencialmente también pueden ser útiles para proponer la resolución de problemas médicos contenidos en los artículos periodísticos, la generación de discusiones en torno a un tema, o conocer el perfil social de las profesiones sanitarias (23).

\section{Conclusiones}

En síntesis, nuestro estudio muestra que:

- en la prensa escrita se encuentran textos referidos a la mayor parte de las enfermedades recogidas en la CIE-10, lo que puede contribuir a la educación para la salud. El tratamiento periodístico de esta información puede ser incidental o mantenido en el tiempo según la naturaleza de las enfermedades;

- la utilización docente de textos periodísticos puede contribuir a la adquisición de competencias transversales en la formación de grado en Medicina y otras Ciencias de la Salud.

Material suplementario: No hay

Financiación: No ha habido financiación

Declaración de conflicto de interés: Los autores declaran no tener ningún conflicto de intereses. 


\section{Referencias}

1. Calvo Hernando M. El periodismo científico, reto de las sociedades del siglo XXI. Comunicar. 2002; 19: 15-8. DOI: https://doi.org/10.3916/25474

2. González Alcaide G, Valderrama Zurián JC, Aleixandre Benavent R. La investigación sobre la divulgación de la ciencia en España: Situación actual y retos para el futuro. Madrid: ARBOR Ciencia, Pensamiento y Cultura. 2009; 738: 861-9. doi: https://10.3989/arbor.2009.738n1058

3. Elías C. Formatos mediáticos de la información científica: prensa, periódicos digitales, radio y televisión. En "Fundamentos del periodismo científico y divulgación mediática". Madrid: Alianza Editorial; 2008, p. 187-217.

4. Semir V. Protagonistas y públicos de la comunicación científica. En: El Científico ante los medios de comunicación. Retos y herramientas para una cooperación fructífera. Barcelona: Fundación Dr. Antonio Esteve; 2013. https://www.esteve.org/libros/cuaderno-irtve/

5. González Silva M. Genes de papel. Genética, retórica y periodismo en el diario El País (1976-2006). Madrid: Consejo Superior de Investigación Científica; 2014. https://editorial.csic.es/publicaciones/libros/12342/0/genes-de-papel-genetica-retorica-yperiodismo-en-e.html

6. González Alcaide G, Valderrama Zurián JC, Aleixandre Benavent R. Análisis del proceso de internacionalización de la investigación española en ciencia y tecnología (1980-2007). Rev Esp Doc Cient. 2012; 35(1): 94-118. DOI: https://10.3989/redc.2012.1.847

7. De Semir V. What is newsworthy? The Lancet 1996; 347: 1.163-6. DOI: 10.1016/s01406736(96)90614-5

8. Lobera J. Percepción social de la ciencia y la tecnología 2016. Madrid: Fundación Española $\begin{array}{llllll}\text { para la Ciencia y la Tecnología, } & \text { FECYT; }\end{array}$ https://www.fecyt.es/es/publicacion/percepcion-social-de-la-ciencia-y-la-tecnologia-enespana-2016

9. Revuelta G, Minelli de Oliva J. La salud y la biomedicina en la prensa diaria. Un análisis de diez años. Periodística. 2008; 55-67. DOI: https://doi.org/10.1157/13086045

10. Won Kim J, Chadha M, Gil de Zuñiga H. El uso de los medios de comunicación y la elaboración cognitiva: el papel mediador de la eficacia mediática. Revista latina de comunicación social. 2018; 73; 168-83. DOI: 10.4185/RLCS-2018-1251

11. Lopera E. entre conversos, escépticos y negacionistas: cómo abordar el análisis de la cobertura informativa de la ciencia del clima. En Díaz García I, Muñoz van den Eynde A (Eds.). Participación y cultura científica en contexto internacional. Madrid: CSIC/Catarata; 2011. Pp. 167-198.

12. Cantabrana B, Diez B, Hidalgo A. Percepción de los estudiantes de la divulgación científica en la prensa y de su contribución a la cultura científica. FEM. 2015; 18: 47-53. DOI: https:// doi.org/10.33588/fem.181.753

13. Cantabrana B, Diez B, Bordallo J, Sánchez M, Hidalgo A. Apropiación de terminología médica por estudiantes de primer curso del grado en Medicina a través de prensa diaria. FEM. 2013; 16 (3): 145-51. DOI: https://doi.org/10.33588/fem.163.692

14. Camacho Markina I. La 'gripe A', en la prensa española. Revista Latina de Comunicación Social. 2009, 64. 827-843. DOI: https://10.4185/RLCS-64-2009-865-827-843.

15. Aiestaran A, Camacho I y Ronco M. La información sobre salud y medicina en los diarios de la Comunidad Autónoma Vasca y Navarra. Ámbitos, 2014; 26: 1-19. http://dx.doi.org/10.12795/Ambitos.2014.i26.12

16. Ronco López M, Peñafiel C y Echegara L. El periodismo de salud en España. La información de salud en la prensa española (2000-2010): aproximación a los estudios documentales existentes. Doc Cien Inf, 2014; 37: 267-304. DOI: http://dx.doi.org/10.5209/rev_DCIN.2014.v37.46827.

17. Revuelta G. Salud y medios de comunicación en España. Gaceta Sanitaria. 2006; 20(1): 203-8. https://www.upf.edu/pcstacademy/_docs/200603 gemma.pdf

18. Wakefield MA, Loken B, Hornik RC. Use of mass media campaigns to change health behavior. Lancet. 2010;376:1261-71. DOI: 10.1016/S0140-6736(10)60809-4. 
19. Robinson MN, Tansil KA, Elder RW et al. Mass media health communication campaigns combined with health-related product distribution: a community guide systematic review. Am J Prev Med. 2014; 47: 360-71. DOI: 10.1016/j.amepre.2014.05.034

20. Martínez Martínez P, Tuells J, Colmenar-Jarillo G. La tardía emergencia mediática de la vacuna contra la viruela, cobertura de noticias en prensa española (1999-2004). Rev Esp Quimioter. 2015; 28:125-31.https://dialnet.unirioja.es/servlet/articulo?codigo $=6255221$

21. Moreno Castro C (Ed.). Campañas institucionales en salud pública: el caso de la vacuna contra el virus VPH. Madrid: Dextra Editorial; 2017.

22. González M, Cantabrana B, Hidalgo A. Ciencia, medicina y medicamentos. Un estudio del periodo 2001-2016. Fundación Educación Médica. 2020; 23:17-24. DOI: 10.33588/fem.231.1034

23. González-García M, Cantabrana Plaza B, Hidalgo Balsera A. propuestas formativas para estudiantes de ciencias de la salud mediante textos periodísticos. Arch Med (Manizales) 2020; 20: 88-99. DOI: https://doi.otg/10.30554/arcmed.20.2.3753

24. Ministerio de Sanidad, Consumo y Bienestar Social. Edición electrónica de la CIE-10-ES Diagnósticos [internet]. Madrid: Ministerio de Sanidad, Consumo y Bienestar Social; 2016 [acceso 18 Mayo 2019]. Disponible en: https://eciemaps.mscbs.gob.es/ecieMaps/browser/index_10 mc_old.html

25. Orden ECI/332/2008, de 13 de febrero, por la que se establecen los requisitos para la verificación de los títulos universitarios oficiales que habiliten para el ejercicio de la profesión de Médico. Boletín Oficial del Estado 2008; 40: 83515.https://www.boe.es/boe/dias/2008/02/15/pdfs/A08351-08355.pdf

26. Kilgo DK, Yoo J, Johnson TJ. Spreading Ebola panic: Newspaper and social media coverage of the 2014 ebola health crisis. Health Commun. 2018; 23: 1-7. DOI: 10.1080/10410236.2018.1437524

27. INE, Instituto Nacional de Estadística. Defunciones según la causa de muerte 2017 [internet]. Madrid: Instituto Nacional de Estadística; [acceso 18 mayo 2019]. Disponible en: http://www.ine.es/jaxi/Tabla.htm?path=/t15/p417/a2017/10/\&file=01001.px\&L=0

28. Benito E. Descubierto un gen responsable de los cánceres de próstata más agresivos. El País, 9 de febrero de 2015. Disponible en: https://elpais.com/elpais/2015/02/09/ciencia/1423497298_393122.html.

29. Méndez M y Morán C. Indemnización para las víctimas de la talidomida 40 años después. El País, 23 de marzo de 2010. Disponible en: https://elpais.com/diario/2010/03/23/salud/1269298803 850215.html

30. Benito E. Los parados se drogan más. El País, 27de enero de 2011. Disponible en: https://elpais.com/diario/2011/01/27/sociedad/1296082804_850215.html

31. Monge Y. EEUU pide perdón a Guatemala por experimentar con pacientes y presos. El País, 2 de octubre de 2010 . Disponible en: https://elpais.com/diario/2010/10/02/internacional/1285970405_850215.html_

32. Benito E. Los enfermos "raros" toman las riendas. El País, 23 de febrero de 2014. Disponible https://elpais.com/sociedad/2014/02/27/actualidad/1393531241_119497.html 